\title{
Comprehensive polarimetric analysis of Spectralon white reflectance standard in a wide visible range
}

\author{
J. M. Sanz, ${ }^{*}$ C. Extremiana, and J. M. Saiz \\ Departamento de Física Aplicada, Universidad de Cantabria, Avda. Los Castros s/n., 39005 Santander, Spain \\ ${ }^{*}$ Corresponding author: sanzjm @unican.es
}

Received 12 April 2013; accepted 25 July 2013;

posted 25 July 2013 (Doc. ID 188788); published 19 August 2013

\begin{abstract}
Since polarimetry has extended its use for the study of scattering from surfaces and tissues, Spectralon, a white reflectance standard, is acquiring the role of a polarimetric standard. Both the behavior of Spectralon as a Lambertian surface and its performance as a perfect depolarizer are analyzed in detail. The accuracy of our dynamic polarimeter, together with the polar decomposition to describe the Mueller matrix (MM) depolarizing action, combine to produce a powerful tool for the proper analysis of this scattering surface. Results allowed us to revisit, for confirmation or revision, the role of some MM elements, as described in the bibliography. The conditions under which it can be considered a good Lambertian surface are specified in terms of incidence and scattering angle and verified over a large wavelength range. (C) 2013 Optical Society of America

OCIS codes: (240.2130) Ellipsometry and polarimetry; (290.5855) Scattering, polarization; (290.1483) BSDF, BRDF, and BTDF.

http://dx.doi.org/10.1364/AO.52.006051
\end{abstract}

\section{Introduction}

Since Spectralon was made up and commercialized by Labsphere Inc. in 1986 [1], it has been used in reflectometry as the closest commercial material to a perfect Lambertian surface. Regardless the angle of scattering, the brightness of a Lambertian surface to an observer is the same. That is why the existence of this kind of surfaces is essential as diffuse reflectance standards, integrating spheres manufacture or high-precision equipment calibration.

Spectralon is composed of polytetrafluoroethylene (PTFE) powder heated and compressed in a special process that conforms a thermoplastic resin. The hardness of this material is similar to that of highdensity polyethylene. It is thermally stable up to temperatures over $350^{\circ} \mathrm{C}$, chemically inert, and hydrophobic. Spectralon has a strong diffuse reflectance, generally more than $99 \%$, over the UV-VisNIR region of the spectrum, and its surface and

$1559-128 \mathrm{X} / 13 / 246051-12 \$ 15.00 / 0$

(C) 2013 Optical Society of America subsurface, structured as a porous network of thermoplastic, exhibit its characteristic reflectance behavior.

Bidirectional diffuse reflectance function (BRDF) of PTFE powder has been studied widely [2] , as well as Spectralon Lambertian behavior for unpolarized light at normal illumination [3]. In the last years, the influence of speckle effects in BRDF laser measurements for the Spectralon has been reported by different authors [4-6] as well as the role of Spectralon from a spectropolarimetric point of view [7-10]. More recently, some researchers have made an effort pursuing a complete polarimetric description of the Spectralon $[11,12]$. All these studies have often been done by giving priority to some polarization parameters, scattering angles, wavelengths or, in many cases, by driving the attention to common reflectometry applications. Since there are several new areas related to the surface polarimetric analysis, such as tissue exploration [13] and oral disease diagnosis [14] in medicine, imaging polarimetry $[15,16]$, or equipment testing [17], mastering the full polarimetric response of surface reflectance standards is an important scientific objective. 
For a scattering system illuminated by a given incident wavelength, the Mueller matrix (MM) is a complete polarimetric result, in the sense that it contains all information about the scattering properties of a system, as far as intensity and polarization of the scattered radiation are concerned. The measurement of the MM is a way for characterizing some features of a scattering system, a part of the so-called inverse problem that is a nonunique process. There has been much work trying to exploit its capabilities by connecting MM properties to those of the scattering system, either optical or geometrical ones $[18,19]$. In the case of highly depolarizing systems, the elements of a MM show a strong mixture of pure and depolarizing properties and some special analysis is required to separate them [20,21]. Handling the polarization states and the $\overline{\mathrm{MM}}$ algebraic properties $[22,23]$, a comprehensive polarimetric description of the Spectralon reflectance standard in a wide visible range can be made. Following this idea, in a recent work [12], authors try to describe a single wavelength $\overline{M M}$ associated to the reflectance of an Spectralon surface, for a range of incidence and scattering angles. They also try to extract information by using a empirical model. As shall be briefly commented later in this work, such a model is not very appropriate because of its strong dependence on both the sample and the experimental setup.

The aim of this research is to make this comprehensive study of the scattering behavior of Spectralon by means of a full polarimetric analysis within the visible spectrum (at 488.0, 520.8, 530.9, 568.2, and $632.8 \mathrm{~nm}$ ) and in a broad range of scattering geometries. First, we want to determine the geometrical conditions, in terms of scattering angle and angle of incidence (AOI), for which Spectralon can be considered a Lambertian surface. Second, we want to examine the quality of Spectralon as a depolarizer within the same conditions. This procedure will provide us with guidelines for the right use of Spectralon not only in reflectance but also in polarimetric measurements.

\section{Experimental Setup}

The dual rotating compensator polarimeter (DRCP) developed in our labs is an improvement of a previous setup [21], in which a multiwavelength source and a pair of high-quality achromatic waveplates have been implemented. It consists of a laser source, a polarization state generator (PSG), composed of a polarizer and a rotating quarter-wave plate, a sample holder, a polarization state analyzer (PSA), composed of a rotating quarter-wave plate and an analyzer, and a detector. A long focal-length lens is placed after the laser and before the PSG in order to control the spot size $(0.6 \mathrm{~mm}$ at the sample location) and beam divergence. A diaphragm, followed by a short focal-length lens, is placed after the PSA and before the detector, to improve both the angular resolution and the overall detector surface illumination. $\mathrm{An} \mathrm{Ar}: \mathrm{Kr}$ laser is able to produce 15 wavelengths in the range from 450 to $568 \mathrm{~nm}$, each of them with a power over $4 \mathrm{~mW}$ and a beam diameter under $0.7 \mathrm{~mm}$. This laser is equipped with a light-feedback regulation system to ensure beam stability. Another laser source (He:Ne laser with an output power of $10 \mathrm{~mW}$ at $\lambda=632.8 \mathrm{~nm}$ ) was used to complete the set of measurements. All the elements between the laser source and the detector are achromatic, from 465 to $640 \mathrm{~nm}$. Quarter-wave plates were previously calibrated over the spectral region of interest, matching the manufacturer specifications and behaving almost identically, something that is important for the correct calculation of the MM with a dynamic polarimeter [24]. Both waveplates rotate synchronously with a speed ratio of $5: 2$, and the joint transmittance describes a Fourier cycle [25] that is related to the polarizer azimuth and the quarter-wave plates azimuthal origin and true retardance. This Fourier cycle can be used to obtain the complete MM of a scattering sample (either transmitting or reflecting), located between the PSG and the PSA, by means of a numerical inverse Fourier transform. The PSA + Detector couple is mounted on the arm of an automated rotor to select the scattering angle, which cover a range from $\theta=-90^{\circ}$ to $\theta=90^{\circ}$, measured from the surface normal and taken positive toward the forward quadrant, except for a blind region of $\pm 20^{\circ}$ around the backscattering direction. The angular step between successive measurements is taken in multiples of $0.5^{\circ}$, adequate for an estimated angular resolution of $0.25^{\circ}$. The sample is on a rotating stage in order to control the AOI (defined positive and measured from the surface normal). The sample holder is on a tilt rotating platform with a XYZ nanopositioner, in order to allow for an accurate alignment as well as a good angular precision $\left( \pm 0.1^{\circ}\right)$. Figure 1 shows a schematic vision of the DRCP (a) and the scattering geometry (b).

A complete set of calibration measurements was done for each laser wavelength. The calibration process consists of a set of 200 measurements, in order to complete a cycle with all possible combinations of the PSG and PSA systems. This procedure is identical for each experimental measurement shown in this work, and produces redundant polarimetric information ready to be processed by an inverse Fourier transform algorithm.

Table 1 shows a no-sample transmission measurement for four wavelengths $(488.0,520.8,568.2$, and $632.8 \mathrm{~nm}$ ). For all five wavelengths analyzed in the range from 488.0 to $632.8 \mathrm{~nm}$, the experimental error associated to the MM elements is kept smaller than $1 \%$ [21], as can be noticed by observing the small deviations with respect to the Identity $4 \times 4$ matrix $\left(I_{4 \times 4}\right)$ in the Table 1 .

The sample analyzed in this work is a certified Spectralon standard (SRS-99 [1]), provided by Labsphere Inc. All measurements were taken inplane. After testing the physical reliability by the Cloude's coherency criterion [26] (a very important, and often neglected check), it was found that all 

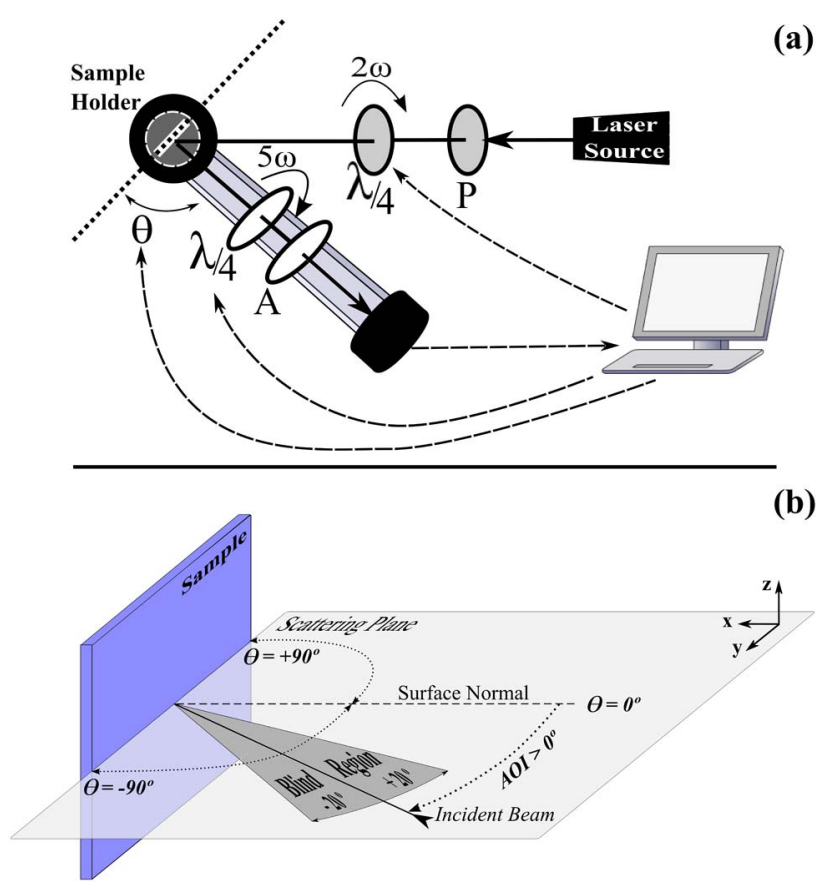

Fig. 1. (a) Experimental setup: positioning (incidence on sample, detector arm, synchronous waveplates rotations) and measurements are computer-controlled. (b) Scattering geometry.

the experimental MMs were reliable and the MM analysis is justified in all the cases analyzed.

\section{Mueller Matrix Interpretation}

The knowledge about a system comes not only from an accurate measurement of its MM, but also from its correct analysis. A common procedure is the MM decomposition (i.e., expressing it as a product-or sum-of several matrices). This is a good option for understanding Spectralon surface scattering, because of its highly depolarizing behavior, a property that can be accounted for under such procedure by means of a specific matrix. For instance, polar decomposition (PD) [22], and in particular forward PD [23], introduces three matrices in the following way:

$$
M=M_{\Delta} M_{R} M_{D}
$$

representing independent physical actions of the system on the polarimetric properties of the scattered light, respectively: $M_{D}$ (diattenuation), $M_{R}$ (retardation), and $M_{\Delta}$ (depolarization), in their operating order [27]. If it is proved that a particular feature of the system is related to any of the parameters contained in such matrices, a direct way of analysis is then opened. Since the PD is an MM algebraic transformation, all results obtained can be applied to conventional polarimetry through an adequate manipulation of the MM elements, $m_{i j}$. It is worth remembering here that forward-PD is a choice among other possibilities $[28,29]$. Because we don't have a system in which some of its physical constituents act sequentially or spatially inhomogeneously on the incoming light, forward-PD may be simple enough and sufficient for this analysis.

For the diattenuation matrix [22], $M_{D}\left(t_{1}, t_{2}, \alpha, \beta\right)$, parameters $\alpha$ and $\beta$ are the azimuth and ellipticity of the diattenuator principal axis, respectively, and $t_{i}$ is the transmittance through the $i$ principal axis. The retardation matrix, $M_{R}(\phi, \delta, \rho)$, contains a phase parameter, $\delta$ (representing the ability of the system to introduce some phase retardation between both orthogonal components or retarder eigenstates), an azimuthal parameter, $\phi$ (that represents the eigenstates orientation origin), and the angular parameter, $\rho$ (representing the optical rotation $[13,30])$. The last operating matrix, particularly interesting for the purpose of this work, is $M_{\Delta}$ [27]. This matrix informs about the depolarizing processes taking place within the system, mostly produced by multiple scattering or other incoherent processes [20].

By introducing the total transmittance (hereafter reflectance, because of the system geometry) for unpolarized incident light $\left(m_{11}\right.$ element of $\left.\mathrm{MM}\right)$, $M_{\Delta}$, can be expressed as

$$
\begin{aligned}
M_{\Delta}\left(m_{11}, d_{i}, a_{i}, z_{i}\right) & =m_{11}\left(\begin{array}{cccc}
1 & 0 & 0 & 0 \\
z_{1} & d_{1} & a_{1} & a_{2} \\
z_{2} & a_{1} & d_{2} & a_{3} \\
z_{3} & a_{2} & a_{3} & d_{3}
\end{array}\right) \\
& =m_{11}\left(\begin{array}{cc}
1 & \mathbf{0}^{T} \\
\mathbf{P}_{\Delta} & m_{\Delta}
\end{array}\right) .
\end{aligned}
$$

Table 1. Four Examples of Typical MMs Obtained with the DRCP for Direct Transmission Measurements at Different Wavelengths (Figures are Rounded to the Significant Digit) ${ }^{a}$

\begin{tabular}{c}
$\quad \lambda=488.0 \mathrm{~nm}$ \\
$\left(\begin{array}{cccc}1.000 & 0.000 & 0.000 & -0.002 \\
-0.001 & 1.000 & -0.002 & 0.002 \\
0.000 & -0.001 & 1.001 & 0.009 \\
-0.001 & 0.001 & 0.001 & 0.998\end{array}\right)$ \\
$\left(\begin{array}{cccc}1.000 & 0.000 & 0.000 & 0.000 \\
0.003 & 1.002 & 0.001 & 0.000 \\
0.000 & 0.000 & 1.001 & 0.003 \\
-0.001 & 0.000 & 0.003 & 1.000\end{array}\right)$ \\
\hline
\end{tabular}

${ }^{a}$ The small differences with respect to the ideal case $\left(I_{4 \times 4}\right.$ matrix) give an idea of the accuracy of the method. 
Nine depolarization parameters appear in $M_{\Delta}$; three of them $\left(a_{i}\right)$ are symmetric and closely associated with retardance, another three $\left(z_{i}\right)$ are related to polarizance, $\mathbf{P}_{\Delta}$, i.e., the polarization capability of $M_{\Delta}$ for unpolarized light, and the three diagonal parameters $\left(d_{i}\right)$, are referred to as principal depolarization parameters [27]. For an ideal Lambertian surface, an isotropic homogeneous depolarizer, only the total reflectance for unpolarized light, $m_{11}$, should differ from 0 .

Finally, it is worth remembering here two basic, but often forgotten, features of MMs, that are particularly important when such matrices have been experimentally obtained. First is the Cloude's coherency criterion [26], a condition to be satisfied by a MM in order to be physically reliable. Second, the degree of polarimetric purity of a physical MM, $P(M)$ $[31, \underline{32}]$, must satisfy the condition $0 \leq P(M) \leq 1$ :

$$
P(M)=\sqrt{\frac{\operatorname{Tr}\left(M^{T} M\right)-m_{11}^{2}}{3 m_{11}^{2}}} .
$$

$P(M)=0$ corresponds to a completely depolarizing MM, while $P(M)=1$ means a pure MM. Whereas calculated MMs are pure $(P(M)=1)$, unless some kind of incoherent averaging is made on the system (time, incident or scattering wavevectors, wavelength, etc.), experimental MMs are intrinsically partially unpolarized.

\section{Results and Analysis}

Two basic reflectance configurations are analyzed in this work: normal and oblique illumination. In both cases the polarimeter has a blind angular window when detecting around the backscattering direction. This is a common feature in reflectance measurements produced by the detection system, by the illumination system or even by both of them, depending on the particular arrangement of each experiment. This problem can be solved by some reflectometer designs $[33,34]$, but its implementation is not accessible for our polarimetric technique. For normal incidence, the angular distribution of the scattered light is symmetric for any scattering plane, and the blind window is located around the surface normal. For oblique incidence, however, patterns are no longer symmetrical when observing within the plane of incidence, and the center of the shadowed region, always in the backscattering direction, moves away from the surface normal (see Fig. 1).

Analyzing the Lambertian behavior of a surface involves, first of all, direct comparison of $m_{11}$ and $\cos (\theta)$, independently of the AOI. In order to simplify this fit, $m_{11}$ has been normalized. Ideally it should be normalized to its value in the direction perpendicular to the surface. For AOI $>20^{\circ}$ such value is available and the normalized value of the reflectance is obtained according to the following weighted expression, in which three values are used in order to reduce the effect of the noise:

$$
\begin{aligned}
& \left.m_{11}(\theta)\right|_{\text {norm }} \\
& \quad=\frac{m_{11}(\theta)}{0.15 m_{11}(-\Delta \theta)+0.7 m_{11}\left(0^{\circ}\right)+0.15 m_{11}(+\Delta \theta)} .
\end{aligned}
$$

For $\mathrm{AOI} \leq 20^{\circ}$ (including normal incidence) such value lies in the blind region, and we have chosen to directly fit the $m_{11}$ plot to a cosine, in the interval of available values $\left[-90^{\circ}, 90^{\circ}\right]$, thus producing a criterion almost identical to the previous one. MM elements are normalized to its corresponding $m_{11}$ element, a common procedure in MM data management.

\section{A. Normal Illumination}

Figure 2 shows the typical behavior of the MM elements for normal illumination $\left(\mathrm{AOI}=0^{\circ}\right.$ ) as a function of the scattering angle $(\theta)$ for $\lambda=520.8 \mathrm{~nm}$ (Lambertian diffuser is plotted in red over the $m_{11}$ values). In Fig. 3 the behavior of element $m_{11}$ is shown for all analyzed wavelengths. These plots are close to their Lambertian curves, with a deviation smaller than $5 \%$ in all cases, unlike the results shown in [12], where deviations of $m_{11}$ were apparently much larger.

We summarize these results in Eq. (ㅁ), where only the $m_{i j}$ elements with significant deviations from zero are printed (let's remember that the perfect depolarizer case corresponds to $m_{i j}=0$, except for $\left.m_{11}\right)$. The rest of $m_{i j}$ elements, averaged over all scattering angles, are zero within the relative error, $<1 \%$, and this stands for the spectral interval between $\lambda=488.0 \mathrm{~nm}$ and $\lambda=568.2 \mathrm{~nm}$. For all MM elements, which noise observed in the angular variation corresponds to the angular size of the speckle, that is not completely averaged by the collecting system because of the high angular resolution imposed on the measuring system (this contribution will be analyzed in depth in Section 4.C). Elements with a systematic average clearly different from zero are $m_{22}=0.05 \pm$ 0.06 and $m_{23} \simeq m_{32}=0.03 \pm 0.06$, all positive, and $m_{12}=-0.02 \pm 0.05$ and $m_{34}=-0.04 \pm 0.05$, that are negative. For $\lambda=632.8 \mathrm{~nm}$ at $\mathrm{AOI}=0^{\circ}$ another element is different from zero, $m_{41}=-0.03 \pm 0.03$. The significance of these nonzero elements can be analyzed either directly from their effect on the scattered light, depending on the state of polarization of the incident light, or indirectly, by means of a forward-PD performed on the MM:

$$
M=m_{11}\left(\begin{array}{cccc}
1 & m_{12} & 0 & 0 \\
0 & m_{22} & m_{23} & 0 \\
0 & m_{32} & 0 & m_{34} \\
0 & 0 & 0 & 0
\end{array}\right) .
$$

\section{Incident Light-Based Analysis}

Except for $m_{11}$, all of the nonzero elements become inactive for incident unpolarized light (first column is the only one acting on a Stokes vector of the form 

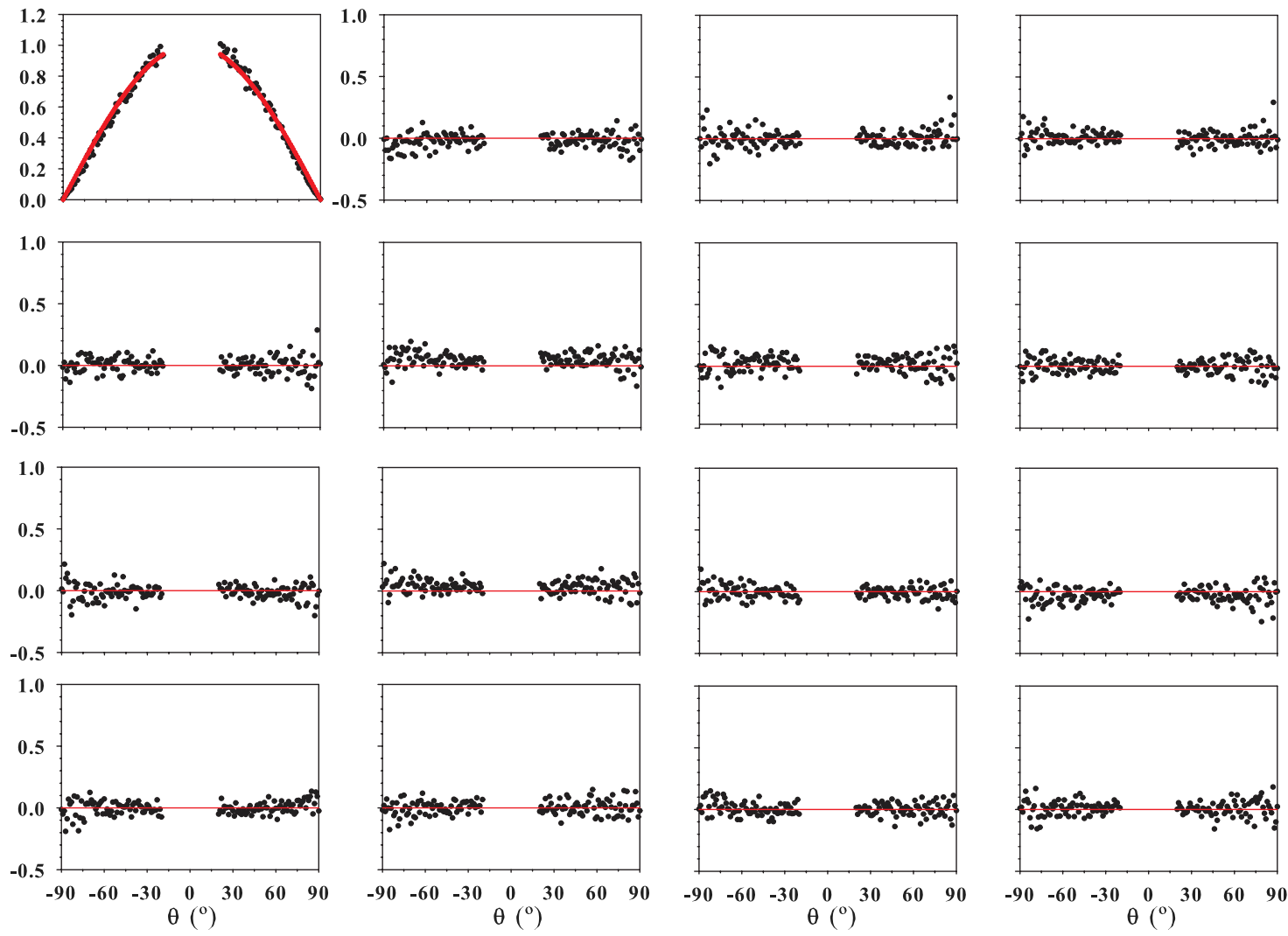

Fig. 2. Black dots: MM elements behavior versus scattering angle $(\theta)$ for Spectralon SRS-99 standard at normal illumination $(\lambda=520.8 \mathrm{~nm})$. Red: Lambertian diffuser behavior.

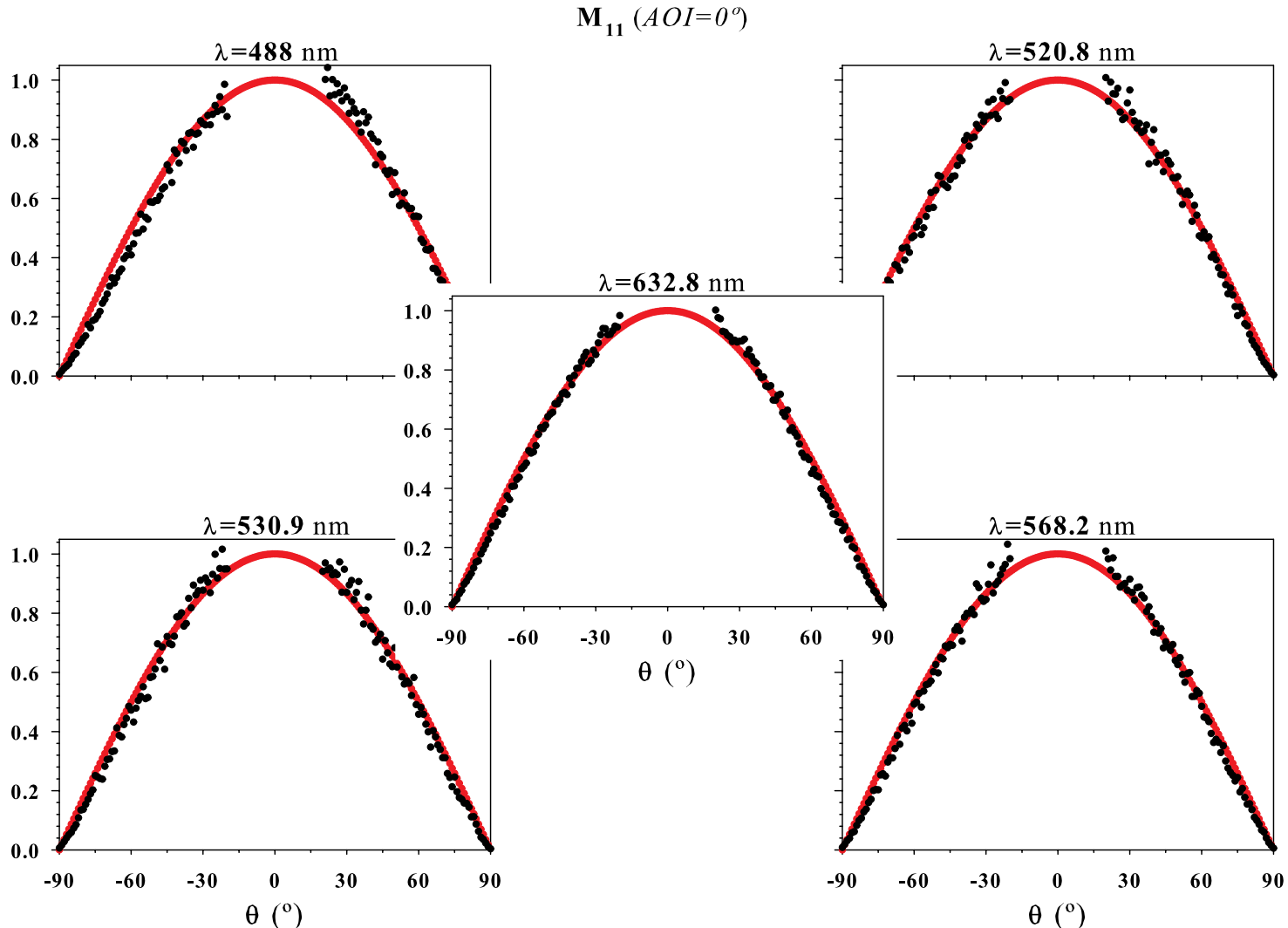

Fig. 3. Black dots: $m_{11}$ element behavior versus scattering angle $(\theta)$ for Spectralon SRS-99 standard at normal illumination $(\lambda=488.0$, $520.8,530.9,568.2$, and $632.8 \mathrm{~nm})$. Red: Lambertian diffuser behavior. 
$\left\langle\begin{array}{llll}1 & 0 & 0 & 0\end{array}\right\rangle^{T}$ ), but for polarized light the small nonzero elements have some particular effects:

(i) If the electric field oscillates parallel ( $p$ polarized) or perpendicular ( $s$-polarized) with respect to the scattering plane (Stokes vector equal to $\left\langle\begin{array}{llll}1 & \pm 1 & 0 & 0\end{array}\right\rangle^{T}$, respectively), the reflectance of a Lambertian surface should not be able to distinguish between both polarization states. However, elements different from zero in Spectralon's MM indicate that, under polarized illumination, BRDF is polarization dependent in proportion to those elements. The influence of $m_{22}, m_{32}$ and $m_{12}$ (in this order) is nonnegligible and, in particular, element $m_{12}<0$ affects intensity selective absorption by introducing diattenuation (the surface scatters more $s$ - than $p$-polarized light). Because of that, BRDF-sscopolarized (BRDF-ss) light measurements should be different from BRDF-pp ones, and also for crosspolarized light ones, as it is known [1, $\underline{9}]$, in connection with the elements in the MM second column. In our case, in the wavelength range from 488.0 to $632.8 \mathrm{~nm}$, BRDF-ss (or BRDF-sp) should be higher than BRDF-pp (or BRDF-ps, respectively), in good agreement with results shown in [12] at $\lambda=530.9 \mathrm{~nm}$. Moreover, in the spectral range analyzed BRDF function verifies that BRDF-ss > BRDF- $p p>$ BRDF-sp > BRDF-ps. Though this result agrees reasonably with previous research $[\underline{7}, \underline{8}]$, it shows a slight change in Spectralon polarimetric behavior as the wavelength goes shorter than $488.0 \mathrm{~nm}$.

(ii) When incident light is linearly polarized out of $s$ or $p$ axis, element $m_{23}$ becomes important. For instance, for $\pm 45^{\circ}$ oriented linear-polarized light (Stokes vector equal to $\left\langle\begin{array}{llll}1 & 0 & \pm 1 & 0\end{array}\right\rangle^{T}$, respectively), it transforms an amount of linear out-ofaxis-polarized light into linear $p$ or $s$-polarized.

(iii) Even if incident light is circularly polarized, $m_{34}$ avoids a Spectralon Lambertian behavior. Left-hand (right-hand) circularly polarized light scatters into linearly $+(-) 45^{\circ}$-polarized light proportionally to this element.

These cases serve as a summary of the Spectralon response to the incident polarization, although obviously many others could be chosen.

\section{Polar Decomposition Analysis}

General considerations on structure and symmetry can be very useful. For instance, the symmetric behavior of $m_{32}$ and $m_{23}$ has been understood as the contribution of a rotated dichroic retarder [12]. However, this interpretation is not always correct being limited to only a few values of rotation and dephase that are able to reproduce such result. Concerning the matrix symmetry and structure shown in Eq. (5) they are fundamentally related to depolarizing activity [both diagonal $\left(d_{i}\right)$ and offdiagonal $\left(a_{1}\right)$, see Eq. (2)], and dichroic activity. Only both effects together are able to generate the Eq. (5) matrix appearance:

$$
\left.M\right|_{0^{\circ}}=\left.\frac{M(\theta)}{m_{11}(\theta)}\right|_{0^{\circ}}=\left(\begin{array}{cccc}
1 & -0.030 & 0 & 0 \\
0 & 0.050 & 0.030 & 0 \\
0 & 0.030 & 0 & -0.040 \\
0 & 0 & 0 & 0
\end{array}\right),
$$

$$
\begin{aligned}
& \left.M\right|_{0^{\circ}}=M_{\Delta} M_{\text {Pure }}=\left(\begin{array}{cccc}
1 & 0 & 0 & 0 \\
0.002 & 0.057 & 0.014 & 0 \\
0.001 & 0.014 & 0.048 & 0 \\
0 & 0 & 0 & 0
\end{array}\right) \\
& \times\left(\begin{array}{cccc}
1 & -0.030 & 0 & 0 \\
-0.024 & 0.786 & 0.574 & 0.230 \\
-0.012 & 0.391 & -0.172 & -0.904 \\
0.014 & -0.480 & 0.800 & -0.360
\end{array}\right) \text {, }
\end{aligned}
$$

$$
\begin{aligned}
\left.M\right|_{0^{\circ}}= & M_{\Delta} M_{R} M_{D}=\left(\begin{array}{cccc}
1 & 0 & 0 & 0 \\
0.002 & 0.057 & 0.014 & 0 \\
0.001 & 0.014 & 0.048 & 0 \\
0 & 0 & 0 & 0
\end{array}\right) \\
& \times\left(\begin{array}{ccccc}
1 & 0 & 0 & 0 & \\
0 & 0.785 & 0.575 & 0.230 \\
0 & 0.391 & -0.172 & -0.904 \\
0 & -0.480 & 0.800 & -0.360
\end{array}\right) \\
& \times\left(\begin{array}{cccc}
1 & -0.030 & 0 & 0 \\
-0.030 & 1 & 0 & 0 \\
0 & 0 & 1 & 0 \\
0 & 0 & 0 & 1
\end{array}\right) .
\end{aligned}
$$

In what follows, we have proceeded under a more transparent scheme, i.e., by performing the forwardPD of the experimental MM. Equations (6a)-(6c) show an example of forward-PD over an average $\mathrm{MM}\left(\mathrm{AOI}=0^{\circ}, \lambda \in[488.0,568.2] \mathrm{nm}\right)$ with threedecimal precision. Equation (6a) shows the average MM within that spectral range, Eq. (6b) shows forward-PD as a product of a depolarizing MM $\left(M_{\Delta}\right)$ and a pure MM ( $\left.M_{\text {Pure }}\right)$, and Eq. (6c) includes the development of $M_{\text {Pure }}$ in two separate parts: the retarding matrix $\left(M_{R}\right)$ and the diattenuation matrix $\left(M_{D}\right)$. The resulting polarimetric elements are a linear diattenuator with its principal axis aligned with the scattering plane direction and its normal $\left(M_{D}\left(t_{1}=0.485, t_{2}=0.515, \alpha=0^{\circ}, \beta=0^{\circ}\right)\right)$ followed by an elliptical retarder [27] $\left(M_{R}\left(\phi=7.14^{\circ}\right.\right.$, $\left.\delta=248.90^{\circ}, \rho=8.34^{\circ}\right)$ ) and a depolarizer. Even though MM asymmetry appears hardly ever in the bibliography, recent works show results reporting matrix asymmetry of $m_{12}$ and $m_{34}$ elements in Spectralon measurements for different wavelengths and geometric configurations [11,12]. PD analysis reveals that asymmetry in $m_{12} / m_{21}$ and $m_{34} / m_{43}$ is not due 


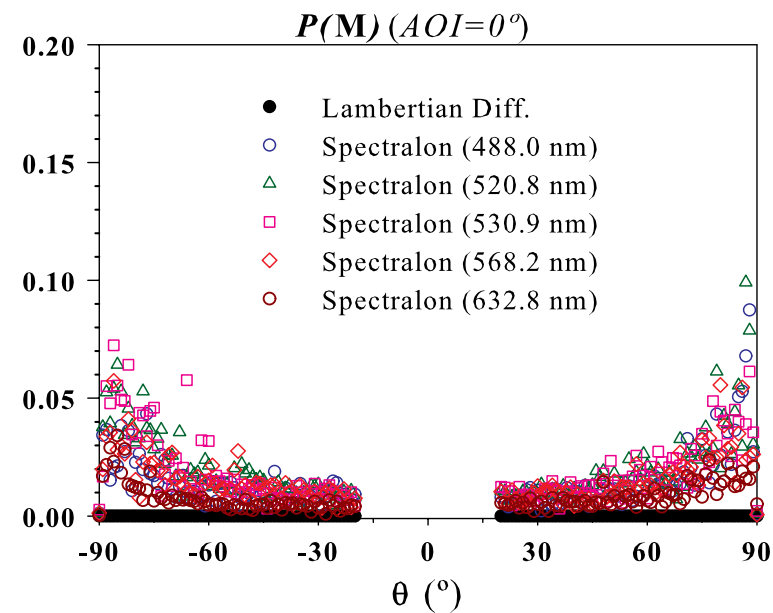

Fig. 4. $\quad P(M)$ for Spectralon SRS-99 standard at normal illumination. Black dots, Lambertian diffuser; blue circles, $\lambda=488.0 \mathrm{~nm}$; green triangles, $\lambda=520.8 \mathrm{~nm}$; pink squares, $\lambda=530.9 \mathrm{~nm}$; red diamonds, $\lambda=568.2 \mathrm{~nm}$; dark-red circles, $\lambda=632.8 \mathrm{~nm}$.

to depolarization activity but due to pure activity: $m_{12}$ responds to $M_{R}$ and $M_{D}$ interplay and $m_{34}$ react to $M_{R}$ azimuth and optical activity [see Eq. (6c)]. On the other hand, symmetry in $m_{23} / m_{32}$ is caused by both diagonal and nondiagonal depolarizing activity [see Eq. (6b)]. Note that MM elements are all null in the last row [see Eq. (6a)], preserving null dephase between light components (i.e., avoiding outgoing elliptically polarized light).

Lambertian behavior of Spectralon can be also analyzed by evaluating the parameter $P(M)$, as defined in Eq. (3). Usually, the smaller the values of $P(M)$, the stronger the depolarizing effect induced on scattered light. Figure 4 shows $P(M)$ of Spectralon in normal incidence for all the analyzed wavelengths, together with the value for an ideal Lambertian surface $(P(M)=0)$. As we can see, $P(M)$ never reaches fully depolarizing values, even when scattering angle is close to backscattering direction. In this case, about $1 \%$ of the scattered light remains polarized, and this quantity increases with the scattering angle, getting over $5 \%$ for grazing observation.

\section{B. Oblique Illumination}

As happened with normal incidence, results were similar for all five wavelengths analyzed under oblique illumination. A complete set of measurements ranging from $\mathrm{AOI}=20^{\circ}$ to $\mathrm{AOI}=85^{\circ}$ in $5^{\circ}$ intervals was performed, of which only MM graphs for $\mathrm{AOI}=40^{\circ}, 75^{\circ}$, and $85^{\circ}$ are shown in Figs. 5, 6, and $\underline{7}$, respectively, for $\lambda=568.2 \mathrm{~nm}$ (but our conclusions should be representative of the general behavior in the spectral range analyzed$\lambda \in[488.0,632.8] \mathrm{nm})$ :
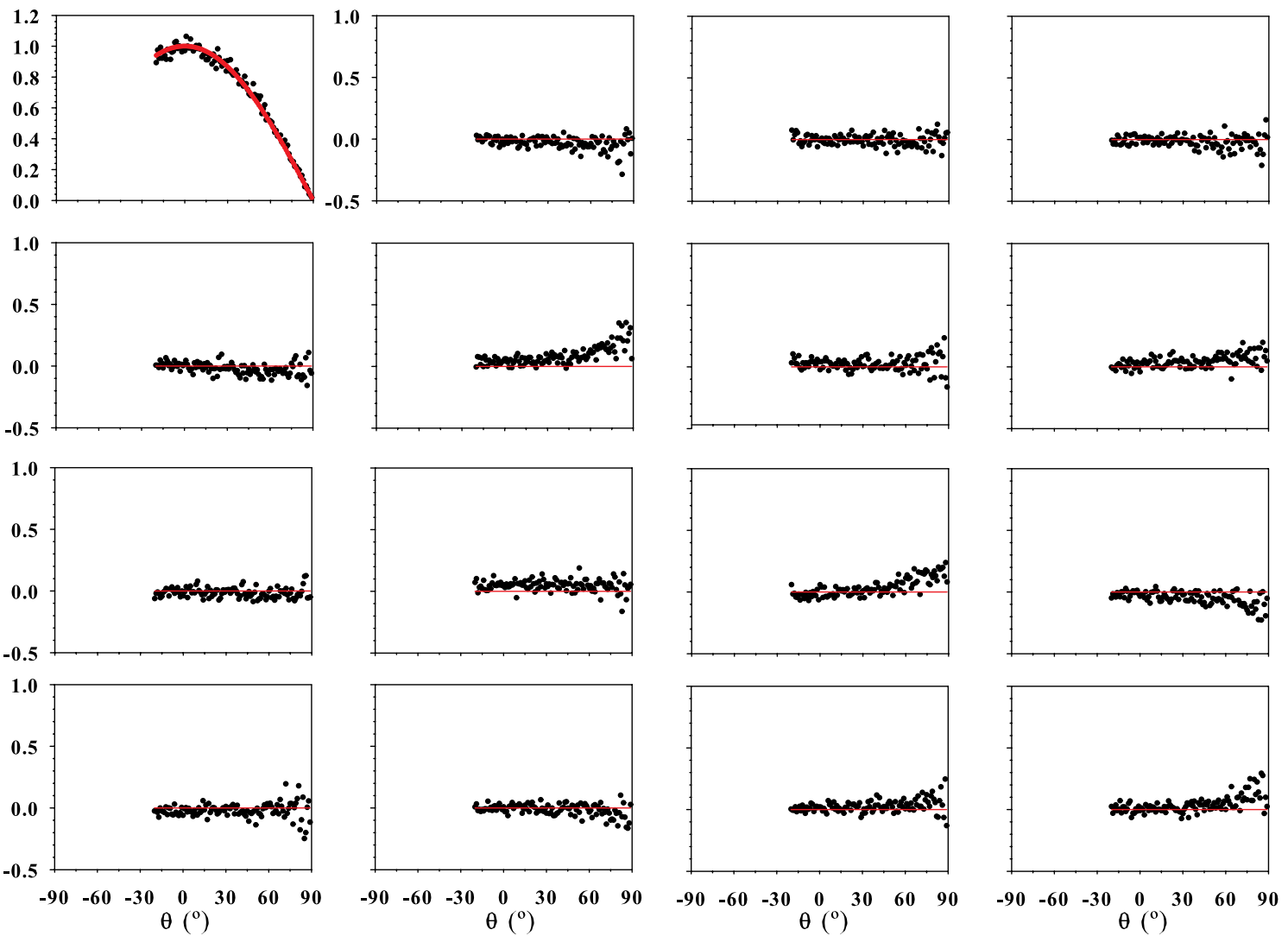

Fig. 5. Black dots: MM elements behavior versus scattering angle $(\theta)$ for Spectralon SRS-99 standard $\left(\mathrm{AOI}=40^{\circ} ; \lambda=568.2 \mathrm{~nm}\right)$. Red: Lambertian diffuser behavior. 


$$
M=m_{11}\left(\begin{array}{cccc}
1 & m_{12} & 0 & 0 \\
\sim m_{12} & m_{22} & \sim 0 & 0 \\
0 & \sim 0 & m_{33} & m_{34} \\
0 & 0 & -m_{34} & \sim m_{33}
\end{array}\right) .
$$

The main point in Fig. 5 is the shape of $m_{11}$, close to that of a Lambertian diffuser (red line) around the surface normal. Obviously, the strongest deviation is found for high values of $\theta$ around the specular (Figs. $\underline{6}$ and $\underline{7}$ ) where the surface coherent scattering is predominant.

As a first approximation, valid for all angles, MM takes the form given by Eq. (7). The off-diagonal $2 \times 2$ boxes of Spectralon's MM are close to zero for all incidences and for all wavelengths, as can be expected in the case of reflection from an isotropic surface [35]. Even though $m_{23}$ and $m_{32}$ elements' average values are around 0.03 , they are very close to being of some significance as a remaining activity. As for the diagonal $2 \times 2$ boxes, they deserve the following comments:

(i) The elements in the diagonal evolve almost identically. While they keep very similar to the normal incidence case for the backward scattering region $\left(\theta<0^{\circ}\right)$, in the forward scattering quadrant $\left(\theta>0^{\circ}\right)$ they increase monotonically with $\theta$. In all cases $m_{22}>m_{33} \geq m_{44}$ and all approach 1 when $\theta$ is close to $90^{\circ}$ for $\mathrm{AOI} \geq 75^{\circ}$. This means that near grazing observation Spectralon actually preserves the incident light polarization.

(ii) Diattenuation and polarizance (given by $m_{12}$ and $m_{21}$, respectively) are very similar. Although they reach a value of just 0.02 in the backscattering region $\left(\theta<0^{\circ}\right)$, both of them evolve toward significant negative values in the forward region $\left(\theta>0^{\circ}\right)$. This behavior is general for AOI $\geq 45^{\circ}$ and the location of the minimum depends on the AOI. Toward grazing observation $m_{12}$ and $m_{21}$ tend to zero again. Thus, the behavior of $m_{12}$ and $m_{21}$ differs from that observed in the normal illumination case, in the same terms that are shown in some references for BRDF measurements [7,8] (BRDF-ss should be higher than BRDF- $p p$ for $\overline{\mathrm{A}} \mathrm{OI} \neq 0^{\circ}$ and $\theta>0^{\circ}$, and they evolve toward an inverse behavior for $\mathrm{AOI} \neq$ $0^{\circ}$ and $\theta<0^{\circ}$, with BRDF-pp higher than BRDF$s s)$. Even for unpolarized light, when AOI $\neq 0$ and $\theta>$ $0^{\circ}$ Spectralon will differ from the Lambertian profile: polarizance element $m_{21}$ will convert unpolarized light into $s$-polarized light. Proportionally to $m_{21}$, the higher the AOI, the greater the amount of $s$-polarized light produced.

(iii) Absolute values of elements $m_{34}$ and $m_{43}$ are quite similar, and its opposite sign make of them an antisymmetric pair, as corresponding to a conventional retarder. The minimum of $m_{34}$ is obtained for $\theta \in\left[50^{\circ}, 60^{\circ}\right]$ and is deeper for AOI $\sim 60^{\circ}$ reaching a value of $m_{34} \simeq-0.15$.
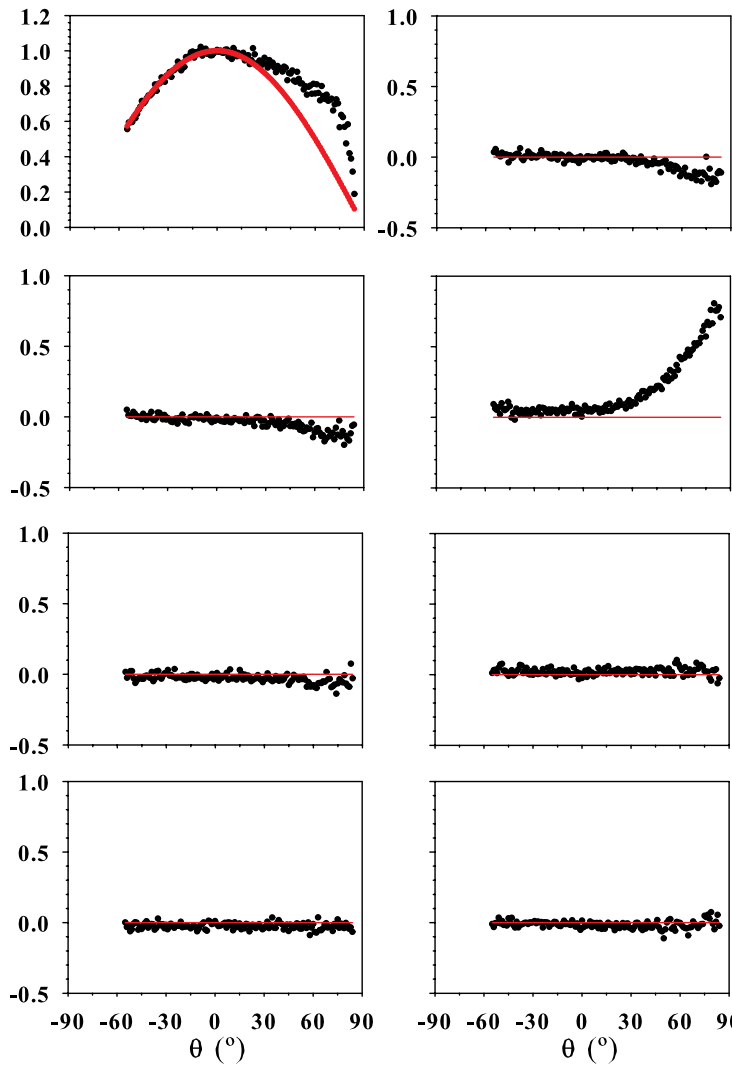
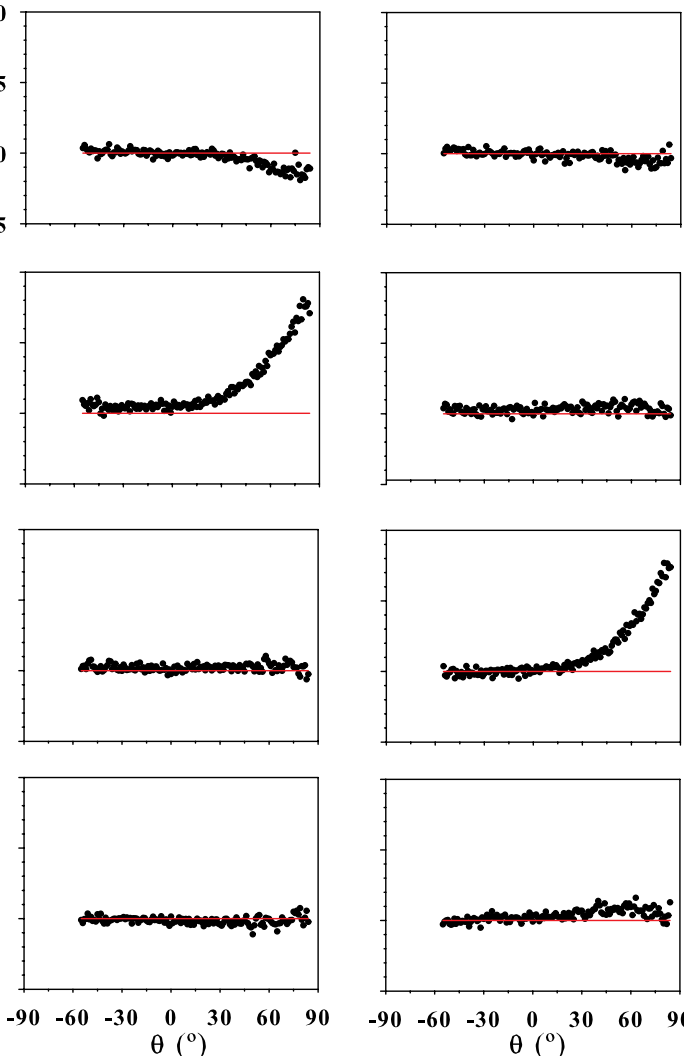
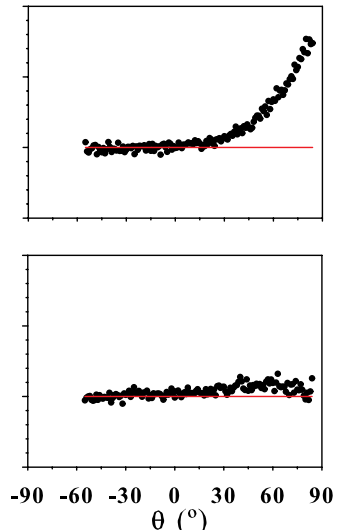
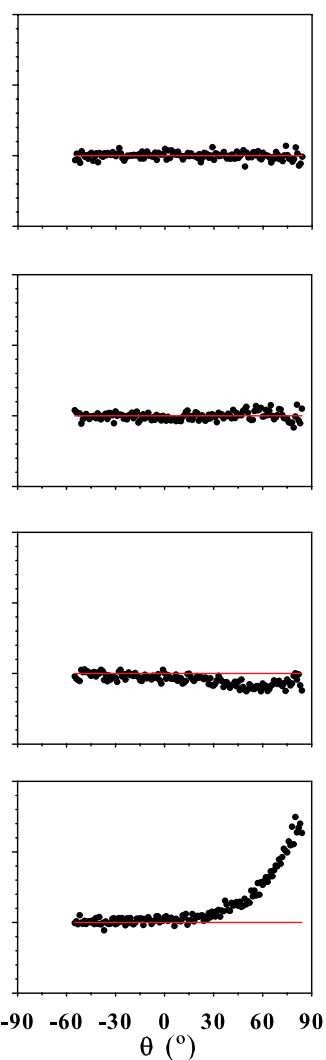

Fig. 6. Black dots: MM elements behavior versus scattering angle $(\theta)$ for Spectralon SRS-99 standard $\left(\mathrm{AOI}=75^{\circ} ; \lambda=568.2 \mathrm{~nm}\right)$. Red: Lambertian diffuser behavior. 

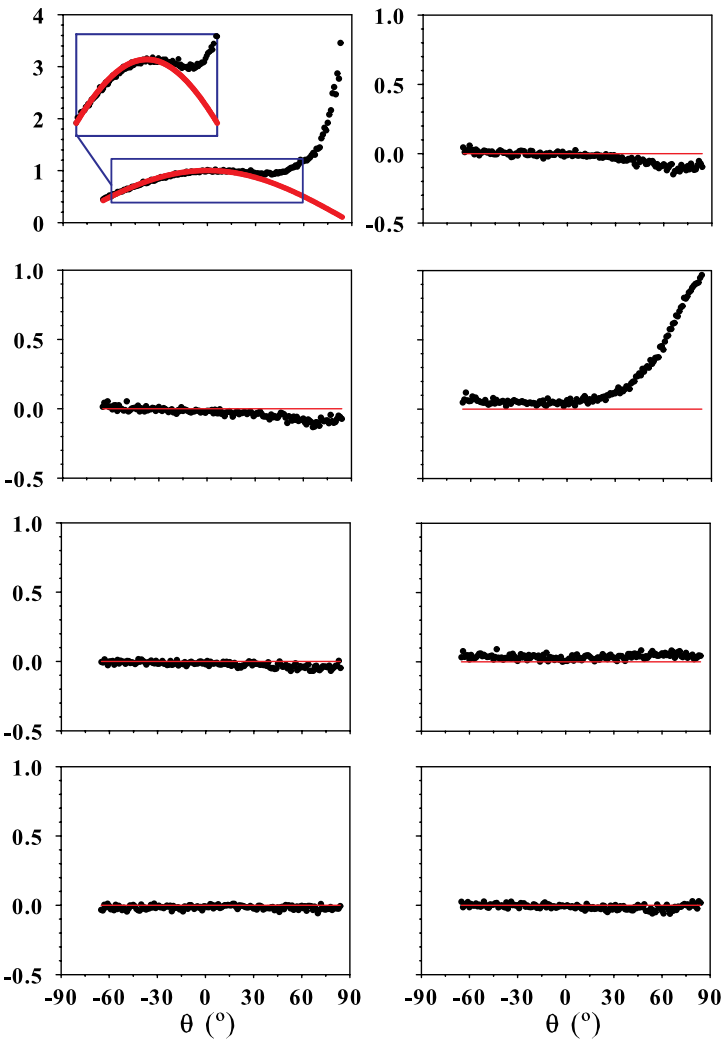

Fig. 7. Black dots: MM elements behavior versus scattering angle $(\theta)$ for Spectralon SRS-99 $\operatorname{standard}\left(\mathrm{AOI}=85^{\circ} ; \lambda=568.2 \mathrm{~nm}\right)$. Red: Lambertian diffuser behavior.

Albeit the remainder elements are close to zero, the couple $m_{23}-m_{32}$ deserves a particular comment. These elements are very close to being of physical significance and, moreover, they are symmetric. If this fact were related to common optical activity (rotation, as suggested in [12]), other off-diagonal elements (such as $m_{24}$ and $m_{42}$ ) would be different from zero. However, $m_{24}$ and $m_{42}$ are near zero, then $m_{23}-m_{32}$ symmetry is not related to optical activity, but to depolarizing diagonal and off-diagonal activity (elements $a_{1}, d_{1}$ and $d_{2}$ in the depolarizing matrix). Figure 8 shows the depolarizing parameters related to the $\bar{M} M$ in Fig. 6 . Though there is a scattering region (nearby $\theta=\overline{\mathrm{A}} \mathrm{OI}$ ) where $a_{1}$ and $z_{i}$ become significant, depolarizing off-diagonal parameters tend to zero for grazing incidence and scattering angles close to $90^{\circ}$. Regarding diagonal elements, $d_{i}$, they are close to zero for $\theta<30^{\circ}$, and they exponentially
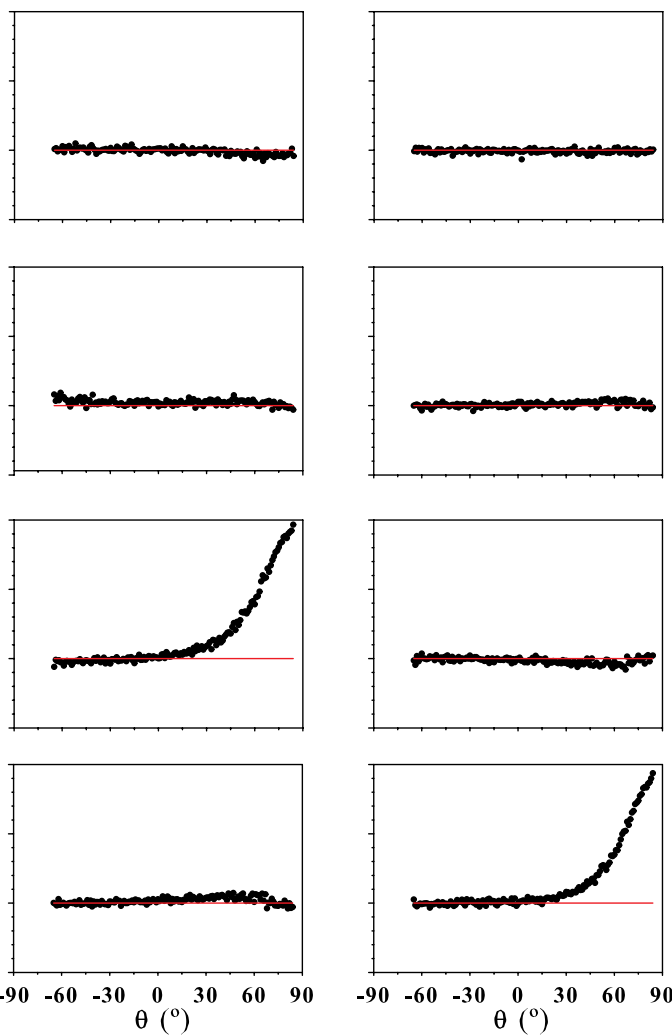
grow for $\theta>30^{\circ}$. Depolarizing matrix turns into Identity matrix for grazing incidence and $\theta \sim 90^{\circ}$.

\section{Speckle Noise Influence}

Although the problem of speckle is specifically addressed by other authors [4], we think it deserves a few comments concerning our own measurements. In order to avoid speckle noise, an average was done over several points in a small region of the sample $\left(\sim 25 \mathrm{~mm}^{2}\right)$ for AOI $=0^{\circ}$ and $\lambda=632.8 \mathrm{~nm}$. Figure 9 shows MM behavior for an average over 5 points. The values are consistent with the description made in Section 4.A for the results shown in Fig. 2. While MM elements averaged over all angles have a mean standard deviation of 0.034 for a single measurement, it reduces to 0.020 and 0.015 for five and ten averaged measurements, respectively. Although speckle noise can be smoothed out by averaging
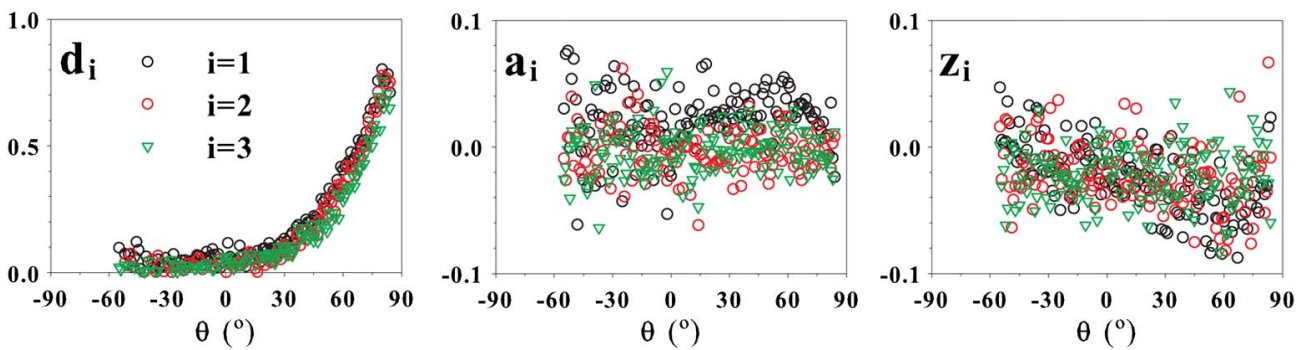

Fig. 8. Depolarization parameters versus scattering angle $(\theta)$ for Spectralon SRS-99 standard (AOI $\left.=75^{\circ} ; \lambda=568.2 \mathrm{~nm}\right)$. Black circles, $i=1$; red circles, $i=2$; and green triangles, $i=3$. 

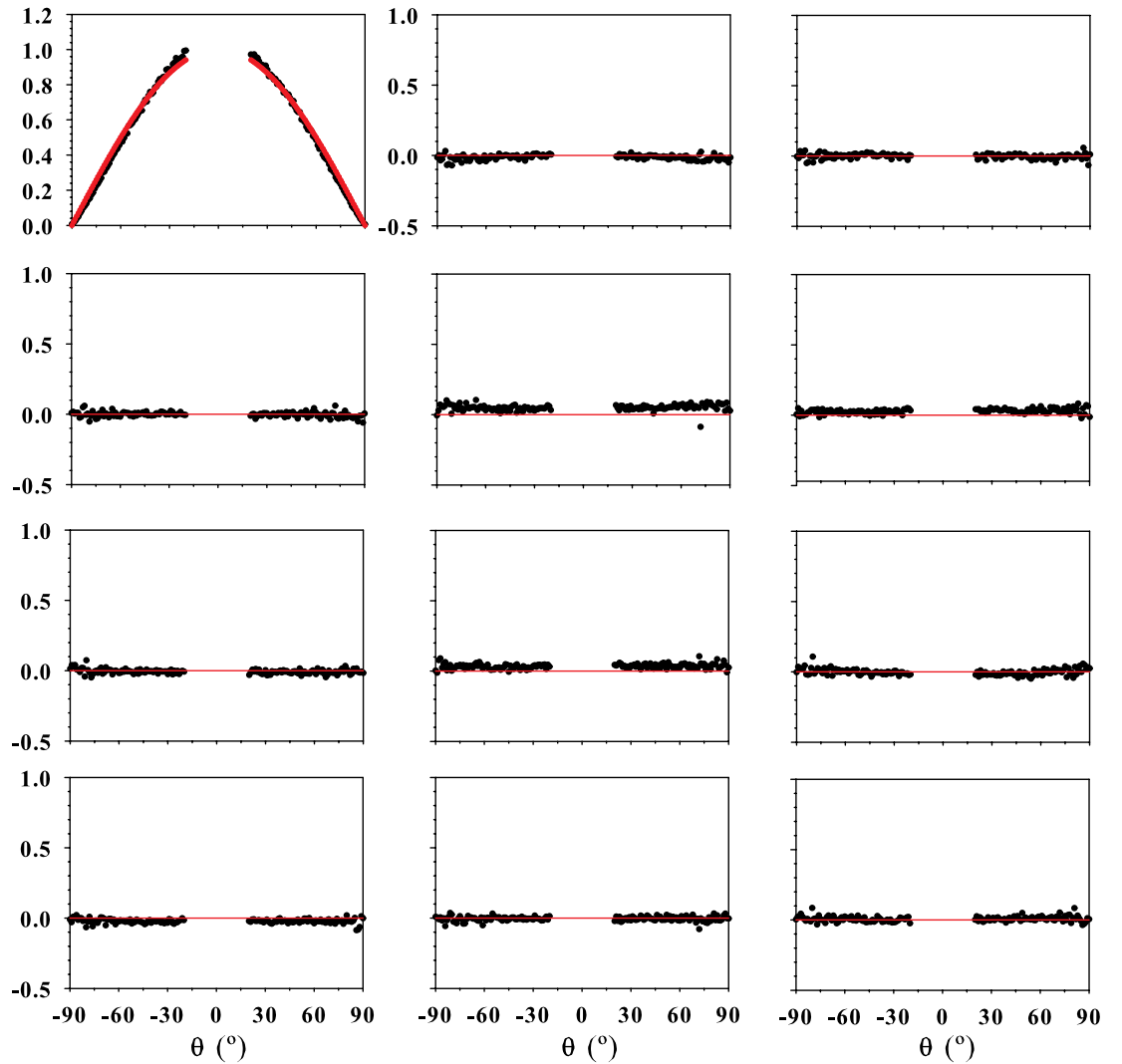
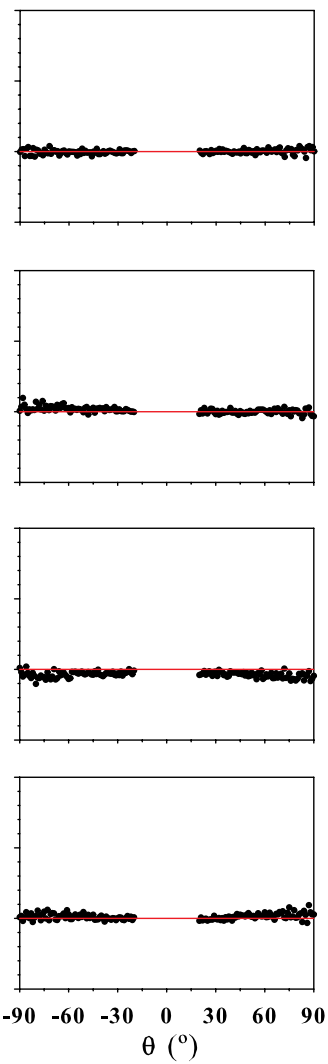

Fig. 9. Black dots: surface-averaged (five positions) MM elements behavior versus scattering angle $(\theta)$ for Spectralon SRS-99 standard at normal illumination $(\lambda=632.8 \mathrm{~nm})$. Red: Lambertian diffuser behavior.

several spots on the Spectralon surface [6], the comments about the significant nonzero average values remain valid.

Other averaging method is that performed on the observation angle. Figure 10 shows the MM behavior after an angular average over $5 \mathrm{deg}$, in a single measurement obtained at normal illumination and $\lambda=632.8 \mathrm{~nm}$. This average is good to describe the potential results obtained for a wider detector, yet it is not a proper way of reducing speckle noise because the nature of this average depends on the speckle size. In our case, speckle size is small enough as to make angular measurements independent from each other, and both averaging methods become very similar (the resulting MM elements averaged over all angles have a mean standard deviation of 0.016).

Finally, it is worth noticing that speckle noise is reduced by increasing AOI, as can be observed in Section 4.B (this is also observed in $[4,36]$ ). This phenomenon is a result of the speckle size reduction produced by the increase in the illuminated surface of the sample.

\section{Lambertian Limits}

The cosine shape of all $m_{11}$ curves is clear for $\theta<0^{\circ}$, as summarized in Fig. 11, while the departure from such Lambertian behavior is obvious for $\theta>0^{\circ}$ and $\mathrm{AOI}>45^{\circ}$. This phenomenon, referred to as off-specular reflection, off-specular maximum, or super-reflection [33], is mentioned in the bibliography and the surface facetting has been pointed out as the cause [7].

Another view of the Lambertian behavior is offered by the degree of polarimetric purity, $P(M)$. This is represented in Fig. 12 in semi-logarithmic scale for several values of $\mathrm{A} \overline{\mathrm{OI}}$ at $\lambda=568.2 \mathrm{~nm}$. As we can see, $P(M)$ differs from zero in the range analyzed. However, $P(M)$ values are around 0.01 in the range $\theta \in\left[-60^{\circ}, 30^{\circ}\right]$ regardless of both AOI and wavelength. In other words, Spectralon SRS-99 bounces off $99 \%$ of fully depolarized light for $-60^{\circ}<\theta<30^{\circ}$ in the wavelength range from 488.0 to $632.8 \mathrm{~nm}$, independently of the AOI $\left(\mathrm{AOI}>0^{\circ}\right)$, outlining a Lambertian reflectance profile. At normal incidence more than $98 \%$ of the reflected light is fully depolarized in the scattering range $-60^{\circ} \leq \theta \leq 60^{\circ}$ (see Fig. 4), with strong deviations when either $\theta \leq-60^{\circ}$ or $\theta \geq 60^{\circ}$. Such deviations increase dramatically for AOI $>45^{\circ}$ and $\theta>30^{\circ}$, resulting in a low depolarizing surface for these conditions.

Since in all cases the experimental reflectance (given by $m_{11}$ ) approaches very well a Lambertian curve (apart from AOI $>45^{\circ}$ and $\theta>30^{\circ}$ ), all comparisons between theoretical models and experimental results should be consistent with this experimental fact. It is surprising to observe in the literature [12] strong deviations from Lambertian behavior for moderate AOI. It may be due to some anomaly in the experimental setup or, more likely, to the logical variability due to quality differences 

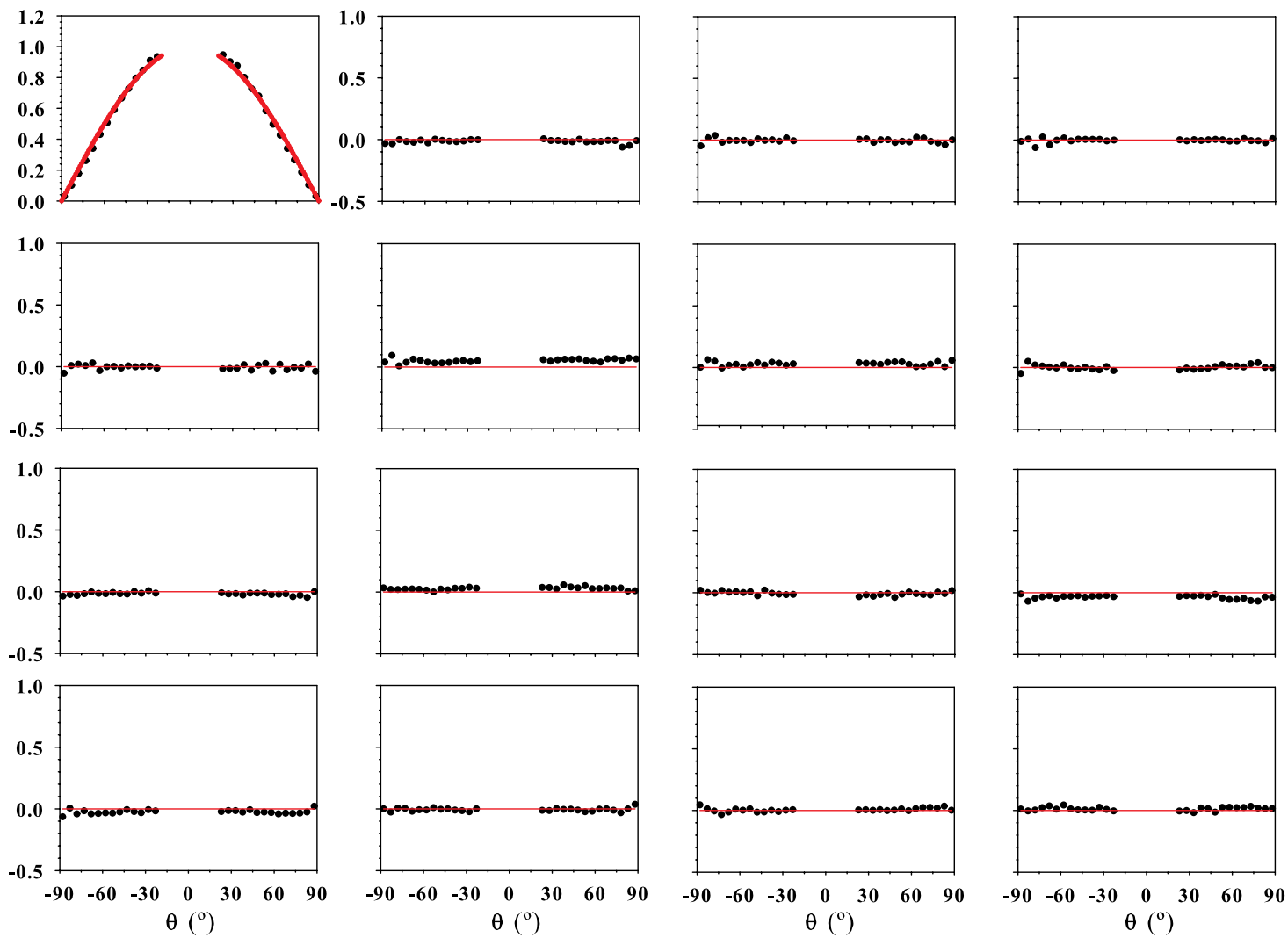

Fig. 10. Black dots: angular averaged (along $5 \mathrm{deg}$ ) $\mathrm{MM}$ elements behavior versus scattering angle $(\theta)$ for a single measurement of Spectralon SRS-99 standard at normal illumination $(\lambda=632.8 \mathrm{~nm})$. Red: Lambertian diffuser behavior.

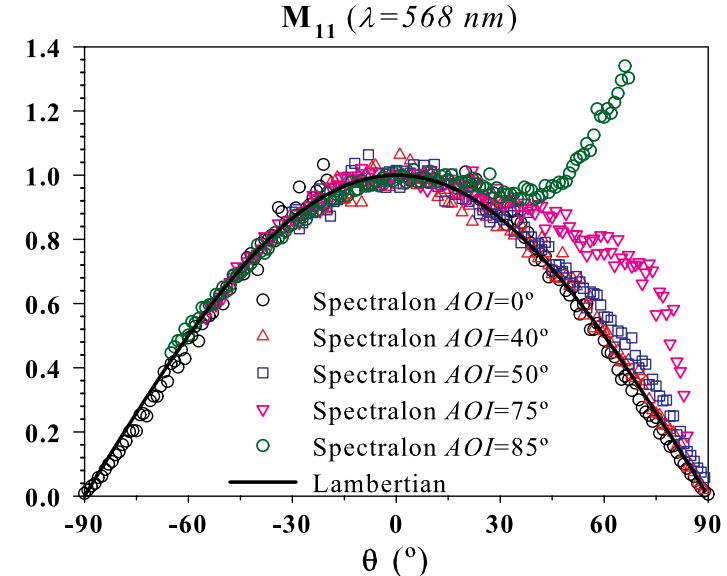

Fig. 11. $m_{11}$ at $\lambda=568.2 \mathrm{~nm}$ versus $\theta$. Black line, Lambertian behavior; black circles, $\mathrm{AOI}=0^{\circ}$; red triangles, $\mathrm{AOI}=40^{\circ}$; blue squares, $\mathrm{AOI}=50^{\circ}$; pink triangles, $\mathrm{AOI}=75^{\circ}$; green circles, $\mathrm{AOI}=85^{\circ}$.

in Spectralon samples. In any case, models that fit the behavior of this standard [11,12] could be helpful in understanding these fault lines.

\section{Final Remarks and Conclusions}

In this research we have done a fully polarimetric analysis of the in-plane light scattered by Spectralon SRS-99 when illuminated with a laser beam. The study was done for illumination geometries ranging

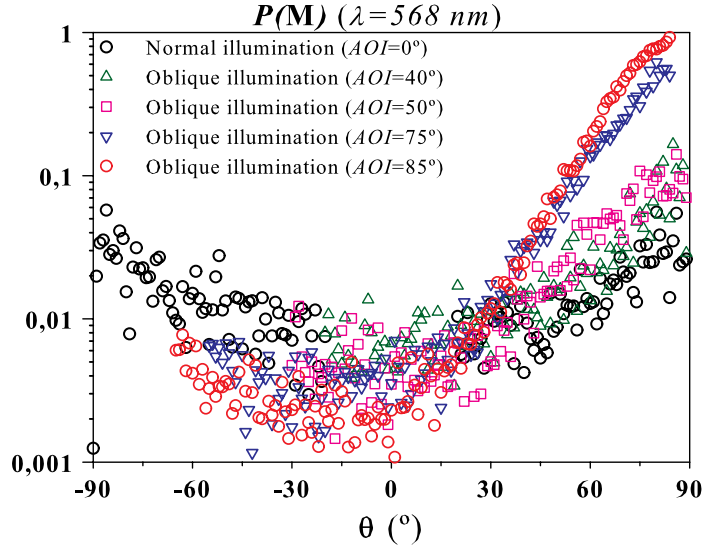

Fig. 12. $\quad P(M)$, in semi-logarithmic scale, for Spectralon SRS-99 standard at some oblique illuminations $(\lambda=568.2 \mathrm{~nm}$. Black circles, $\mathrm{AOI}=0^{\circ}$; green triangles, $\mathrm{AOI}=40^{\circ}$; pink squares, $\mathrm{AOI}=50^{\circ}$; blue triangles, $\mathrm{AOI}=75^{\circ}$; red circles, $\mathrm{AOI}=85^{\circ}$ ).

from $\mathrm{AOI}=0^{\circ}$ to $\mathrm{AOI}=85^{\circ}$, and light wavelengths from 488.0 to $632.8 \mathrm{~nm}$. The effect of the speckle noise on the measurements has been specifically addressed. All the results have been analyzed both by the conventional MM interpretation and by PD. This second method in particular is very helpful to avoid misinterpretations of some of the MM elements that are found in the literature.

In normal illumination, the polarimetric response of Spectralon SRS-99 shows deviations from 
Lambertian as small as $2 \%$ in the range $\theta \in\left[-60^{\circ}, 60^{\circ}\right]$. According to element $m_{11}$, its deviations from the Lambertian regime are also smaller than $2 \%$ (within that range). However, Spectralon is no longer a perfect depolarizer when $|\theta|>60^{\circ}$, with deviations around $10 \%$, as shown in the $P(M)$ plot (see Fig. 4). Furthermore, those deviations increase drastically when Spectralon is analyzed in oblique illumination, AOI $>45^{\circ}$, and detection, $\theta>30^{\circ}$.

With that said, under certain conditions of illumination and detection, Spectralon seems to be not just a good reflectance standard, but a polarimetry standard as well. Regarding that, for oblique illumination $\left(\mathrm{AOI} \neq 0^{\circ}\right)$ in the in-plane scattering region ranging from $\theta=-60^{\circ}$ to $\theta=30^{\circ}$, SRS-99 can be understood as a Lambertian diffuser, as well as a perfect depolarizer, within the same limits as a reflectance standard (99\%), in a wavelength range from 488.0 to $632.8 \mathrm{~nm}$.

Finally, on Spectralon when comparing our results with other previously published, we have noticed remarkable differences concerning its Lambertian character. Being a high quality and widely accepted reflectance standard, a check of the experimental repetitivity of the reflectance curves over a representative set of samples is advisable.

This research has been supported by the Ministry of Education of Spain under project FIS2010-21984.

\section{References}

1. Labsphere Inc., "A guide to diffuse reflectance coatings and materials," http://www.labsphere.com/uploads/technicalguides/a-guide-to-reflectance-materials-and-coatings.pdf.

2. V. R. Weidner and J. J. Hsia, "Reflection properties of pressed polytetrafluoroethylene powder,” J. Opt. Soc. Am. 71, 856-861 (1981).

3. K. J. Voss and D. Zhang, "Bidirectional reflectance of dry and submerged Labsphere Spectralon plaque," Appl. Opt. 45, 7924-7927 (2006).

4. G. T. Georgiev and J. J. Butler, "The effect of speckle on BRDF measurements," Proc. SPIE 5882, 588203 (2005).

5. B. T. McGuckin, D. A. Haner, R. T. Menzies, C. Esproles, and A. M. Brothers, "Directional reflectance characterization facility and measurement methodology," Appl. Opt. 35, 4827-4834 (1996).

6. S. Kaasalainen, E. Ahokas, J. Hyyppä, and J. Suomalainen, "Study of surface brightness from backscattered intensity: calibration of laser data," IEEE Geosci. Remote Sens. Lett. 2, 255-259 (2005).

7. D. A. Haner, B. T. McGuckin, and C. J. Bruegge, "Polarization characteristics of Spectralon illuminated by coherent light," Appl. Opt. 38, 6350-6356 (1999).

8. A. A. Bhandari, B. Hamre, Ø. Frette, L. Zhao, J. J. Stamnes, and M. Kildemo, "Bidirectional reflectance distribution function of Spectralon white reflectance standard illuminated by incoherent unpolarized and planepolarized light," Appl. Opt. 50, 2431-2442 (2011).

9. D. H. Goldstein, D. B. Chenault, and J. L. Pezzaniti, "Polarimetric characterization of Spectralon," Proc. SPIE 3754, 126 136 (1999).

10. D. H. Goldstein and D. B. Chenault, "Spectropolarimetric reflectometer," Opt. Eng. 41, 1013-1020 (2002).

11. T. A. Germer and H. J. Patrick, "Mueller matrix bidirectional reflectance distribution function measurements and modeling of diffuse reflectance standards," Proc. SPIE 8160, 81600D (2011).

12. Ø. Svensen, M. Kildemo, J. Maria, J. J. Stamnes, and $\emptyset$. Frette, "Mueller matrix measurements and modeling pertaining to Spectralon white reflectance standards," Opt. Express 20, 15045-15053 (2012).

13. M. K. Swami, S. Manhas, P. Buddhiwant, N. Ghosh, A. Uppal, and P. K. Gupta, "Polar decomposition of $3 \times 3$ Mueller matrix: a tool for quantitative tissue polarimetry," Opt. Express 14, 9324-9337 (2006).

14. J. Chung, W. Jung, M. J. Hammer-Wilson, P. Wilder-Smith, and Z. Chen, "Use of polar decomposition for the diagnosis of oral precancer," Appl. Opt. 46, 3038-3045 (2007).

15. X. Li and G. Yao, "Mueller matrix decomposition of diffuse reflectance imaging in skeletal muscle," Appl. Opt. 48, 2625-2631 (2009).

16. C. Collet, J. Zallat, and Y. Takakura, "Clustering of Mueller matrix images for skeletonized structure detection," Opt. Express 12, 1271-1280 (2004).

17. M. Smith, "Polarization metrology moves beyond homebrewed solutions," Laser Focus World 40, 123-129 (2004).

18. H. C. van de Hulst, Light Scattering by Small Particles (Dover, 1981).

19. S. N. Savenkov, L. T. Mishchenko, R. S. Muttiah, Y. A. Oberemok, and I. A. Mishchenko, "Mueller polarimetry of virus-infected and healthy wheat under field and microgravity conditions," J. Quant. Spectrosc. Radiat. Transfer 88, 327-343 (2004).

20. M. Foldyna, E. García-Caurel, R. Ossikovski, A. D. Martino, and J. Gil, "Retrieval of a non-depolarizing component of experimentally determined depolarizing Mueller matrices," Opt. Express 17, 12794-12806 (2009).

21. J. M. Sanz, J. M. Saiz, F. González, and F. Moreno, "Polar decomposition of the Mueller matrix: ellipsometric ruleof-thumb for square-profile surface structure recognition," Appl. Opt. 50, 3781-3788 (2011).

22. J. J. Gil and E. Bernabeu, "Obtainment of the polarizing and retardation parameters of a non-depolarizing optical system from the polar pecomposition of its Mueller matrix," Optik 76, 67-71 (1987).

23. S. Y. Lu and R. A. Chipman, "Interpretation of Mueller matrices based on polar decomposition," J. Opt. Soc. Am. A 13, 1106 (1996).

24. M. H. Smith, "Optimization of a dual-rotating-retarder Mueller matrix polarimeter," Appl. Opt. 41, 2488-2493 (2002).

25. R. M. A. Azzam, "Photopolarimetric measurement of the Mueller matrix by Fourier analysis of a single detected signal," Opt. Lett. 2, 148 (1978).

26. S. R. Cloude, "Group theory and polarization algebra," Optik 75, 26-36 (1986).

27. J. J. Gil, "Polarimetric characterization of light and media," Eur. Phys. J. Appl. Phys. 40, 1-47 (2007).

28. R. Ossikovski, A. De Martino, and S. Guyot, "Forward and reverse product decompositions of depolarizing Mueller matrices," Opt. Lett. 32, 689-691 (2007).

29. R. Ossikovski, "Analysis of depolarizing Mueller matrices through a symmetric decomposition" J. Opt. Soc. Am. A 26, 1109-1118 (2009).

30. J. M. Sanz, P. Albella, F. Moreno, J. M. Saiz, and F. González, "Application of the polar decomposition to light scattering particle systems," J. Quant. Spectrosc. Radiat. Transfer 110, 1369-1374 (2009).

31. J. J. Gil and E. Bernabeu, "Depolarization and polarization indices of an optical system," Opt. Acta 33, 185-189 (1986).

32. J. J. Gil, "Characteristic properties of Mueller matrices," J. Opt. Soc. Am. A 17, 328-334 (2000).

33. A. Hope and K. O. Hauer, "Three-dimensional appearance characterization of diffuse standard reflection materials," Metrologia 47, 295-304 (2010).

34. A. M. Rabal, A. Ferrero, J. Campos, J. L. Fontecha, A. Pons, A. M. Rubiño, and A. Corróns, "Automatic goniospectrophotometer for the absolute measurement of the spectral BRDF at in- out-of-plane and retroreflection geometries," Metrologia 49, 213-223 (2012).

35. OSA, Handbook of Optics, Vol. 1, Chap. 14-16 (McGraw-Hill, 1994).

36. D. Rod White, P. Saunders, S. J. Bonsey, J. van de Ven, and H. Edgar, "Reflectometer for measuring the bidirectional reflectance of rough surfaces," Appl. Opt. 37, 3450-3454 (1998). 